\title{
Optimization preparation of the biosynthesis of silver nanoparticles using watermelon and study of itsantibacterial activity
}

\author{
Eman Alzahrani $^{1 *}$, Kevin Welham ${ }^{2}$ \\ ${ }^{1}$ Chemistry Department, Faculty of Science, Taif University, 888-Taif, Kingdom of Saudi Arabia \\ ${ }^{2}$ Analytical Chemistry Group, Department of Chemistry, University of Hull, Hull, UK \\ *Corresponding author E-mail: em-s-z@hotmail.com
}

Copyright () 2014 Eman Alzahrani and Kevin Welham. This is an open access article distributed under the Creative Commons Attribution License, which permits unrestricted use, distribution, and reproduction in any medium, provided the original work is properly cited.

\begin{abstract}
The field of nanotechnology is the most active area of research in modern materials science. There is increased demand on nanoparticles because of their wide applicability in various areas; for example, electronics, catalysis, chemistry, energy and medicine. Commonly, silver nanoparticles are fabricated using toxic and flammable chemicals. Therefore, the aim of this work is to find a cost effective and environment-friendly technique for green synthesis of silver nanoparticles from silver nitrate (AgNO3) solution using watermelon extract. The different parameters were optimised for the fabrication of silver nanoparticles including the contact time, concentration of watermelon extract, concentration of silver nitrate solution, reactant ratio, and reaction temperature. The fabricated nanoparticles were characterised using different instruments such as UV-Vis spectrophotometry and their size determined by transmission electron microscopy (TEM), while the element composition of the sample was obtained from the energy dispersive X-ray (EDX) spectrum. The antibacterial effect of the fabricated silver nanoparticles against two human pathogens Escherichia coli (E. coli) and Staphylococcus aureus (S.aureus) was studied and the obtained results confirm the fact that SNPs have the antibacterial property of nanoparticles.
\end{abstract}

Keywords: Synthesis Silver Nanoparticle; Watermelon Extract; Green Chemistry; Antibacterial Activity.

\section{Introduction}

Application of nano-scale materials, ranging from 1-100 nm, is an emerging area of nanotechnology. Silver nanoparticles have high thermal stability, a high specific surface area, high mechanical strength, and have antibacterial activity [1-3]. Traditionally, different methods can be used for the fabrication of silver nanoparticles such as reduction in solutions, chemicals and photochemical reactions in reverse micelles, thermal decomposition of silver compounds, radiation assisted and microwave assisted processes, and green chemistry [4], [5].

Using chemicals can lead to the formation of certain toxic chemicals that are absorbed on the surface of nanoparticles, and these chemicals have negative effects on medical applications, limiting them [6]. Silver nanoparticles can be environmentally fabricated using plant leaf extract, bacteria, fungi and enzymes [7]. The main benefit of using the green chemistry route for the fabrication of silver nanoparticles is to fabricate a material that will be compatible for pharmaceutical and other biomedical applications since the toxic chemicals are not used during the fabrication of nanoparticles [8]. Moreover, using a plant extract can decrease the cost of preparation and eliminate the need for any special culture preparation and isolation technique compared with using bacteria and fungi for fabrication of nanoparticles [9].

The first time that any plant extract was utilised for the fabrication of nanoparticles was performed by Kothari et al. [1]. They fabricated plant mediated silver nanoparticles using papaya fruit extract, and found that the fabricated particles are highly toxic against different multi drug resistant human pathogens. Since then, a number of plants have been investigated in the fabrication of nanoparticles. Live alfa alfa plants have been used to synthesise nanoparticles with a size range of 2-20 nm [10]. Nanoparticles of silver, nickel, cobalt, zinc and copper have been fabricated using Brassica 
juncea (Indian mustard), Medicago sativa (alfa alfa) and Heliantus annus (sunflower). All of these plants have the ability to accumulate higher concentration of metals compared with other plants [11].

In 2009, Song et al. [12] fabricated nanoparticles using Magnolia kobus and Diopyros kaki leaf extracts, and the effect of temperature on the formation of those nanoparticles was studied. It was found that using lower temperature can form polydisperse particles with a size range of 5-300 nm while using higher temperature can form smaller and more spherical particles.

In 2010, David et al. [13] fabricated silver nanoparticles using the leaf extract of Euphorbia hirta L, as a reducing and capping agent. The results saw the synthesised silver nanoparticles showing antifungal activity against Candida albicans, C.kefyr and A.niger. In 2011, Lee et al.[14] used Saururus chinensis leaf extract to fabricate silver nanoparticles. They found that the silver nanoparticles were formed after one day of reaction time. The IR analysis of the samples showed the involvement of proteins and amino groups in the reduction and stabilisation of the silver nanoparticles.

In 2012, Salem and Awwad [15] fabricated silver nanoparticles by using mulberry leaf extract and found effective antibacterial activity to Staphylococcus aureus and Shigella sp. Ponarulselvam et al. [16] developed a novel method for the fabrication of silver nanoparticles using leaf extract of Catharanthus roseus (C. roseus), and they confirmed activity against the malaria parasite Plasmodium falciparum (P. falciparum). Kumar [17] fabricated silver and gold nanoparticles in the size range of $10 \mathrm{~nm}$ using Parthenium Hysterophorus plant.

The aim of this work is to use an environment-friendly technique for green synthesis of silver nanoparticles using watermelon extract. In addition, this work will investigate the findings relating to optimisation of different experimental parameters, and the antibacterial effect of the fabricated silver nanoparticles against two human pathogens E. coli and S.aureus will be checked.

\section{Experiment}

\subsection{Chemicals and materials}

Watermelon was purchased from a local supermarket - Taif, KSA. Silver nitrate $\left(\mathrm{AgNO}_{3}\right)(99.8 \%)$ was purchased from Sigma-Aldrich (Poole, UK). The Whatman filter paper (pore size $25 \mu \mathrm{m}$ and diam. $15 \mathrm{~cm}$ ) was purchased from SigmaAldrich (Poole, UK). Luria Bertani were used here and supplied by Hi-Media Laboratories (Mumbai, India). Distilled water was employed for preparing all the solutions and reagents.

\subsection{Instrumentation}

A hot-plate stirrer from VWR International LLC (West Chester, PA, US), transmission electron microscopy (TEM) from JEOL Ltd. (Welwyn Garden City, UK), a water bath from PolyScience (Niles, Illinois, US), a UV-Vis spectrophotometer from Thermo Scientific ${ }^{\mathrm{TM}}$ GENESYS 10S (Toronto, Canada). Energy dispersive X-ray (EDX) analysis was performed using an INCA 350 EDX system from Oxford Instruments (Abingdon, UK), and the centrifuge was from Lab Essentials, Inc. (Monroe, Georgia, US).

\subsection{Biosynthesis of silver nanoparticles}

Silver (Ag) nanoparticles were synthesised using watermelon extract as a reducing and capping agent. To prepare the plant solution, $10 \mathrm{~g}$ of watermelon was thoroughly washed with distilled water, cut into fine pieces, and filtered using a Whatman filter paper. The plant extract was added drop by drop to silver nitrate aqueous solution that was placed in a $250 \mathrm{~mL}$ Erlenmeyer beaker while stirring at $700 \mathrm{rpm}$, and then the mixture was kept in a dark place.

\subsection{Optimization of different parameters}

The effects of the reaction conditions such as the contact time, concentration of watermelon extract, concentration of silver nitrate solution, reactants ratio, and reaction temperature were studied to maximise the yield of SNPs. The experiment was performed in triplicate for reproducibility. The resulting solutions (SNPs) were monitored using a UVVis spectrophotometer.

\subsubsection{Contact time}

The effect of the incubation time was studied by monitoring the absorption spectra of silver nanoparticles formed in the reaction media at different durations every $15 \mathrm{~min}$, and the reaction was monitored using UV/Vis spectrophotometer from 0 to $150 \mathrm{~min}$ in order to optimise the time required for the reaction completion. The described procedure was repeated for optimisation of concentration of the plant extract. For this purpose, different concentrations of watermelon extract were utilised $(25 \%, 50 \%, 75 \%$ and $100 \%)$. 


\subsubsection{Concentration of silver nitrate solution}

The above described procedure was performed and different concentrations of silver nitrate aqueous solution were investigated $(0.25 \mathrm{mM}, 0.5 \mathrm{mM}, 1 \mathrm{mM}$, and $2 \mathrm{mM})$ in order to optimise the concentration of silver nitrate solution.

\subsubsection{Ratio of plant extract and silver nitrate}

Different ratios of plant extract and silver nitrate solution were investigated $(5: 25,10: 20,15: 15,20: 10$ and 25:5) in order to find the maximum production of SNPs.

\subsubsection{Temperature of reaction}

The temperature of the reaction was set at $25^{\circ} \mathrm{C}, 45^{\circ} \mathrm{C}, 65^{\circ} \mathrm{C}$ and $85^{\circ} \mathrm{C}$ using the water bath for optimisation of the reaction temperature.

\subsection{Characterization of silver nanoparticles}

\subsubsection{Visual observation and UV-Visible spectra analysis}

The colour of the solution was observed by naked eye in order to check the formation of the silver nanoparticles. The bio reduction of the reaction mixture of pure silver ions during exposure to the extract of watermelon was easily followed by observing the UV-Vis spectrum at different time intervals taking $1 \mathrm{~mL}$ of sample, compared with $1 \mathrm{~mL}$ of distilled water as a blank. The absorbance of the resulting solutions was monitored UV-Vis spectrophotometrically in the range $350-800 \mathrm{~nm}$.

\subsubsection{TEM}

The formation of SNPs was confirmed by using transmission electron microscopy (TEM). For this, $5 \mu \mathrm{L}$ of the sample solution was put onto lacy carbon coated $3 \mathrm{~mm}$ diameter copper grids. TEM images were acquired with a Gatan Ultrascan 4000 digital camera attached to a JEOL 2010 transmission electron microscope running at $20 \mathrm{kV}$.

\subsubsection{EDAX}

The fabricated silver nanoparticles were separated by centrifuging at $1100 \mathrm{rpm}$ for $15 \mathrm{~min}$. Energy dispersive X-ray (EDAX) analysis was utilised to get the chemical composition of the fabricated materials.

\subsection{Antibacterial activity study}

The antibacterial effect of the fabricated SNPs was investigated on human pathogenic E. coli and S. aureus using the standard disc diffusion method. A Luria Bertani (LB) broth/agar medium was utilised to cultivate bacteria. Sterile paper discs (diam. $5 \mathrm{~mm}$ ) of silver nitrate solution, watermelon extract, silver nanoparticles $\left(100 \mu \mathrm{g} \mathrm{mL}{ }^{-1}\right)$ were placed on plates besides ciprofloxacin. Then the plates were incubated at $37^{\circ} \mathrm{C}$. After 24 hours, the plates were checked for zones of inhibition (clear areas) and the diameters of the zones were measured using a meter ruler and expressed in millimetres.

\section{Results and discussion}

\subsection{Optimization fabrication of SNPs}

Silver nanoparticles have been used as catalysts, in micro-electronics, and they are extremely effective in medicine. The usual chemical method for synthesising silver nanoparticles involves the use of toxic chemicals such as sodium borohydride. Such methods are expensive, and the toxic chemicals mean that the resulting nanoparticles are noncompatible for human use. More recently, nanoparticles have been fabricated using fungi, bacteria and plant extracts. Commonly, reduction of metal ions using fungi or bacteria needs a longer incubation time, while using plant extracts can reduce the metal ions in a much lesser time. Green chemistry has advantages over chemical and physical methods since it is cheap, environment-friendly, and does not need to use high pressure, energy, temperature or toxic chemicals. Moreover, plants have been used to fabricate nanoparticles since they are readily available, safe to handle, and they have a broad variability of metabolites that can aid in reduction [9], [18]. Hence, we aim to use environmentally friendly and greener methods to synthesise silver nanoparticles. In this work, watermelon extract was used for synthesis 
of silver nanoparticles, and different parameters for fabrication of SNPs were investigated for maximum SNP production.
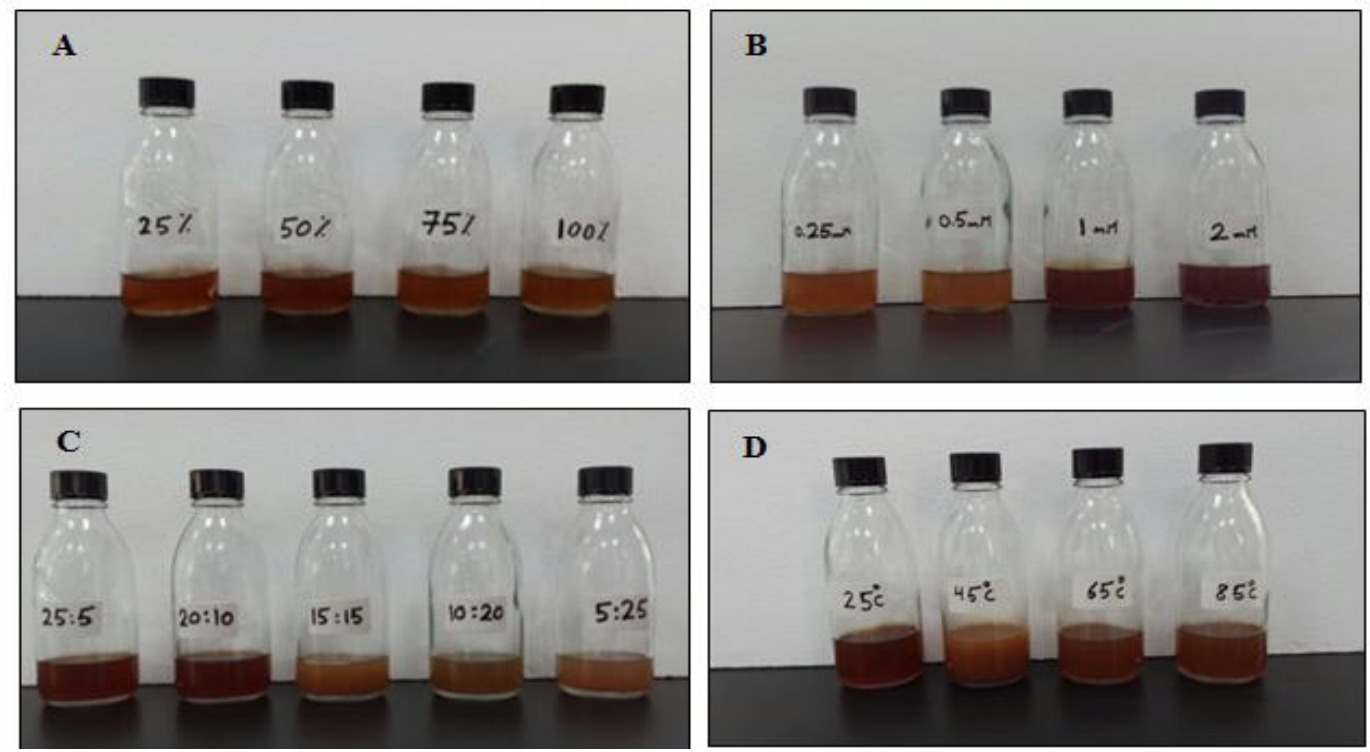

Fig. 1: The Pictures Show the Colour Changes after the Process of Reduction of $\mathrm{Agno}_{3}$ to Ag Nanoparticles (A) Concentration of Watermelon Extract, (B) Concentration of Silver Nitrate Solution, (C) Ratio of Silver Nitrate Solution to Watermelon Extract, and (D) Effect of Temperature.

The colour of the solution was observed by naked eye in order to check the formation of the silver nanoparticles. It was found that the colour of the aqueous solution of $\mathrm{AgNO}_{3}$ changed from transparent to brown when adding the plant extract, as can be seen in Figure 1, which presents photographs showing the changing colour of the solution using different parameters for fabrication of SNPs. The reason for the colour change arises from the reduction of silver ions. In addition, the progress of reduction of silver nitrate reaction within metal ions in the presence of watermelon extract can be easily evaluated by UV-Vis spectra of silver nanoparticles in aqueous solution using a UV-Visible spectrophotometer. This is because silver nanoparticles can exhibit optical properties directly related to Localised Surface Plasmon Resonance (LSPR), which is based on the morphology of the nanoparticles. The UV-Vis spectrograph of the colloidal solution of silver nanoparticles was recorded as a function of time with distilled water as a blank from 350 to $800 \mathrm{~nm}$.

\subsubsection{The contact time}

The first factor considered for optimization of the fabrication of SNPs was the incubation time. The absorption spectra of silver nanoparticles formed in the reaction media at different durations every 15 min were studied. Figure 2 shows the UV-Vis spectra of reduction of silver ions to silver nanoparticles using watermelon extract at different reaction times.

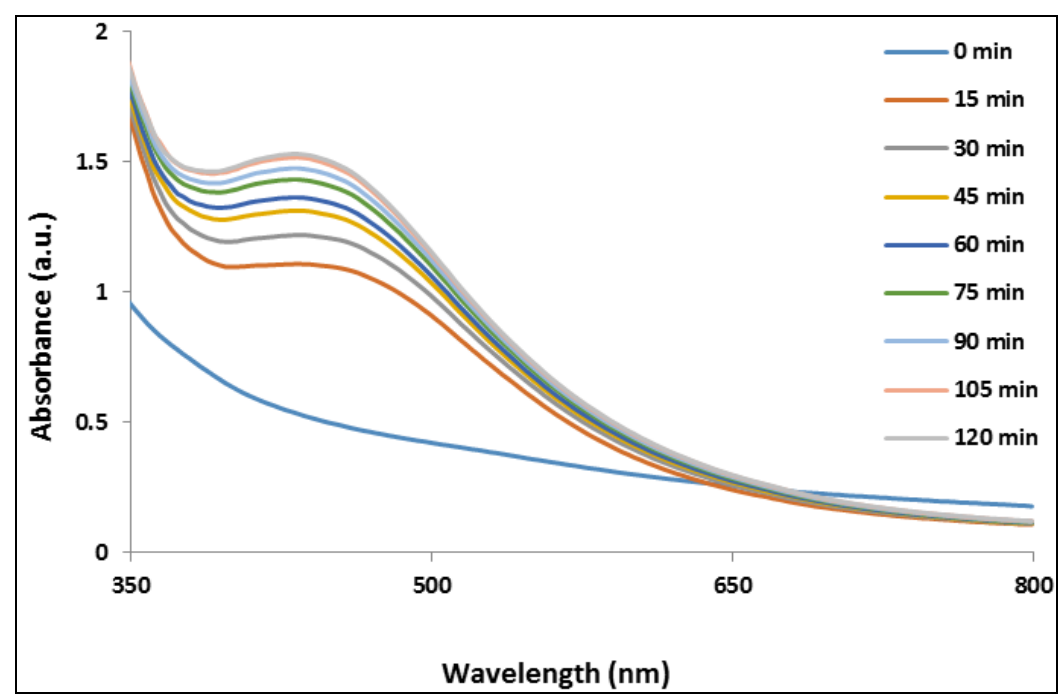

Fig. 2: UV-Vis Absorption Spectra Recorded As Function of Time for Silver Nanoparticles after Bio reduction $\mathrm{Agno}_{3}$ Using Watermelon Extract. 
It was found that when the incubation time was less than 15 min there was no formation of SNPs because the redox potential of the silver nitrate is reduced. Moreover, it was observed that by increasing the time of reaction, the absorption peak was increased, and more SNPs were formed during the study incubation time range (0-120 min). From the spectrum, it was found that the optimum incubation time for the completion of reaction in this study was 120 min. In addition, it was observed that increasing the incubation time to more than 120 min did not increase the absorption significantly, which indicates the stability of the SNP colloidal solution.

\subsubsection{Concentration of watermelon extract}

After discovering the optimum contact time, the concentration of the watermelon extract was investigated and different concentrations of watermelon extract $(25 \%, 50 \%, 75 \%$ and $100 \%)$ were utilised for the maximum production of SNPs and to make sure that reduced silver nitrate was present in the solution. The effect of the watermelon concentration on the fabrication of silver nanoparticles was evaluated spectrophotometrically. After $120 \mathrm{~min}$, the reduction of silver ions was monitored by UV-Visible spectrum analysis of the reaction medium. As can be seen in Figure 3, the highest absorption peak was observed when using $100 \%$ watermelon extract, indicating that the most favourable concentration of watermelon extract of $100 \%$ is quite precise for fabrication of SNPs by watermelon extract.

\subsubsection{Concentration of silver nitrate solution}

The next factor investigated was the concentration of the $\mathrm{AgNO}_{3}$ solution. Different concentrations of $\mathrm{AgNO}_{3}$ solution $(0.25 \mathrm{mM}, 0.5 \mathrm{mM}, 1 \mathrm{mM}$ and $2 \mathrm{mM})$ were utilised in order to maximise the yield to SNPs, and the absorbance of the silver colloidal solution was monitored using the UV-Visible spectrophotometer. As can be seen in Figure 4, the UVVisible absorption spectra showed that by increasing the concentration of silver nitrate solution, the absorbance of SNPs was amplified, and the maximum yield of SNPs was obtained when the concentration of $\mathrm{AgNO}_{3}$ solution was $2 \mathrm{mM}$.

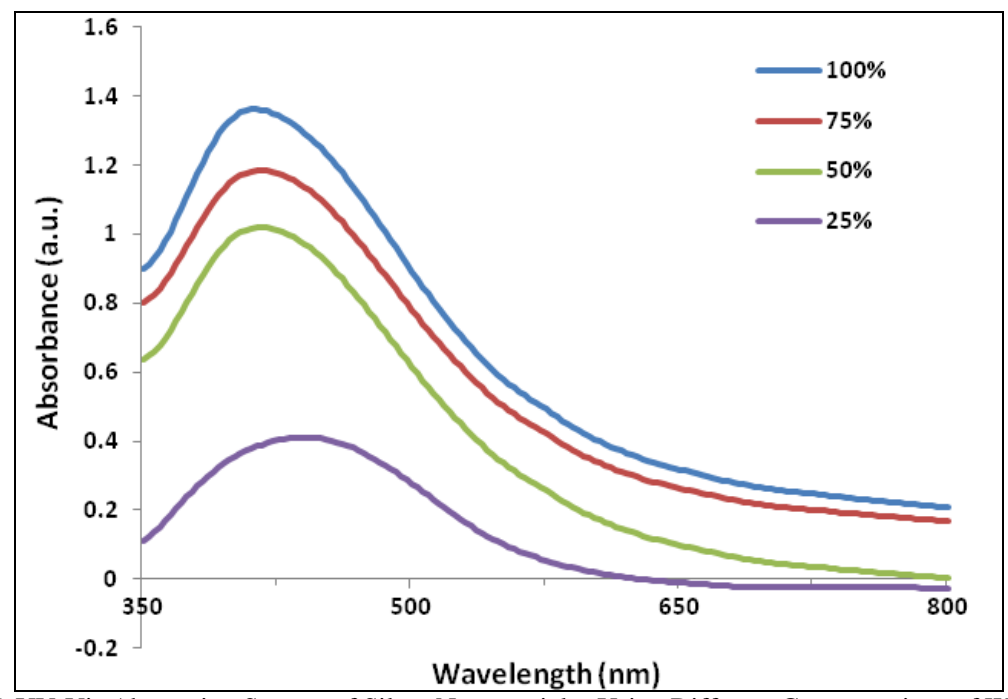

Fig. 3: (A) UV-Vis Absorption Spectra of Silver Nanoparticles Using Different Concentrations of Watermelon.

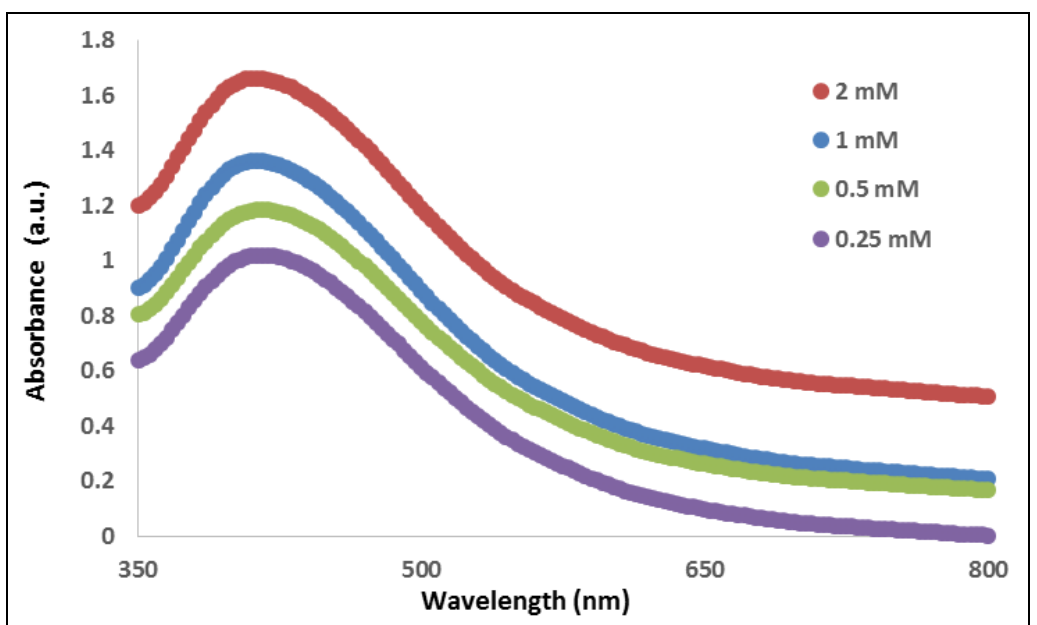

Fig. 4: UV-Vis Absorption Spectra of Silver Nanoparticles Using Different Concentration of $\mathrm{Agno}_{3} \mathrm{Solution}_{\text {. }}$ 


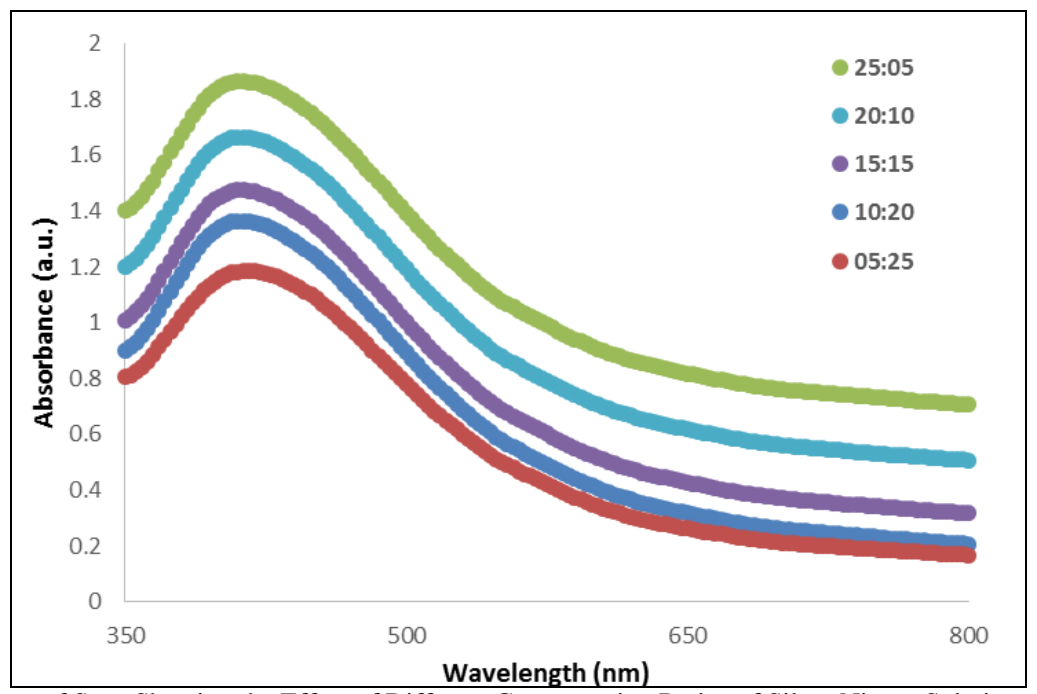

Fig. 5: UV-Visible Spectra of Snps Showing the Effect of Different Concentration Ratios of Silver Nitrate Solution and Watermelon Extract.

\subsubsection{Concentration ratio of watermelon extract and silver nitrate solution}

The concentration ratios of watermelon extract and silver precursor in the ranges of 5:25, 10:20, 15:15, 20:10 and 25:5 were utilised in order to find out the optimum composition of the preparation of SNPs since reactants ratio is an important aspect affecting SNP fabrication and symmetrical nanoparticles preparation. From the results (Figure 5), it was found that the optimum composition to get a high yield of SNPs is when the ratio of silver nitrate solution and watermelon extract in the reaction mixture is 25:5 based on the number of trials and the optimum yield.

\subsubsection{Temperature of reaction}

The last investigated parameter for the fabrication of SNPs is temperature of reaction, which is an important factor since it controls the nucleation process of nanoparticle configuration. The temperature of the reduction reaction was studied by repeating the abovementioned procedure while the reaction temperature was maintained at $25^{\circ} \mathrm{C}, 45^{\circ} \mathrm{C}, 65^{\circ} \mathrm{C}$ and $85^{\circ} \mathrm{C}$ using the water bath, and the absorbance of the resulting solution was measured spectrophotometric ally. Figure 6 shows that as the temperature of reaction increased, the rate of formation of SNPs also increased; however, it was found that increasing the temperature of the reaction to $85^{\circ} \mathrm{C}$ caused the growth of crystals around the nucleus, resulting in a decrease in absorption. An increase in the yield of SNPs was observed when the temperature of the reaction was increased from $25^{\circ} \mathrm{C}$ to $65^{\circ} \mathrm{C}$, though beyond this there was a fall in absorbance.

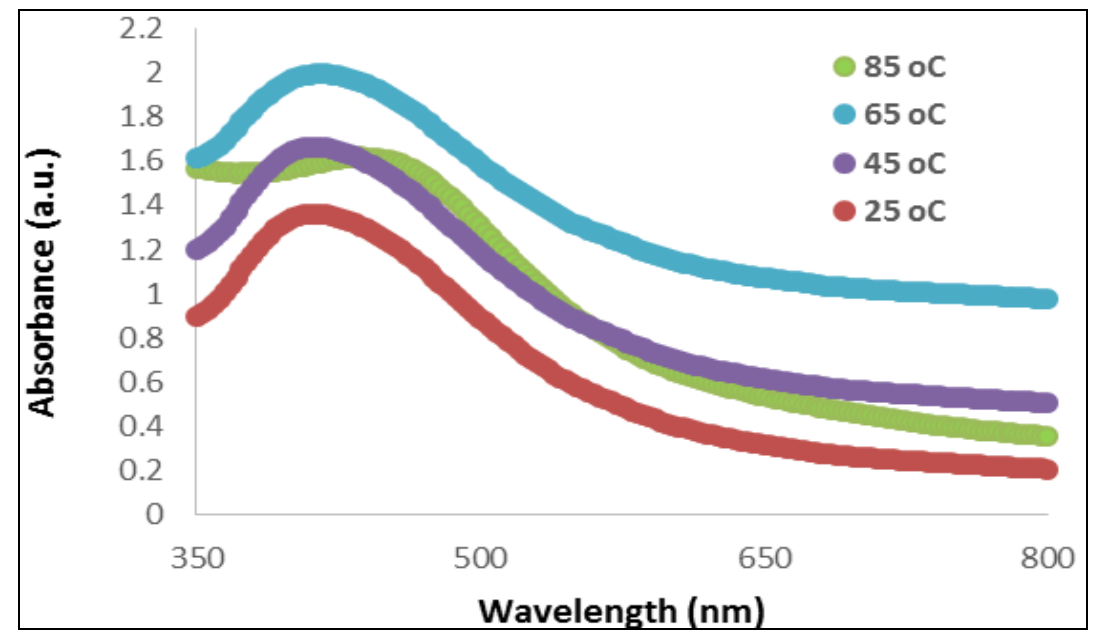

Fig. 6: UV-Vis Absorption Spectra of Silver Nanoparticles Showing Effect of Reaction Temperature

Based on the results, the overall optimised reaction condition was as follows: the incubation time was 120 min, the concentration of watermelon extract was $100 \%$, the concentration of silver nitrate solution was $2 \mathrm{mM}$, the ratio of silver nitrate solution and watermelon extract was $25: 5$ and the temperature of the reaction was $65^{\circ} \mathrm{C}$. It was found that the fabricated silver nanoparticles were stable up to 1 month at room temperature. 


\subsection{Characterization of SNPs}

In this study, the optimized synthesized SNPs were characterized using TEM, which can provide information about the morphology and size of fabricated nanoparticles. Figure 7 presents the TEM micrographs of the bio fabricated silver nanoparticles, using different magnifications, which shows that fabricated SNPs were well dispersed without aggregation, possessing a spherical shape. In addition, it was found that the particle size was $14 \mathrm{~nm}$ with a standard deviation of $6 \mathrm{~nm}$.
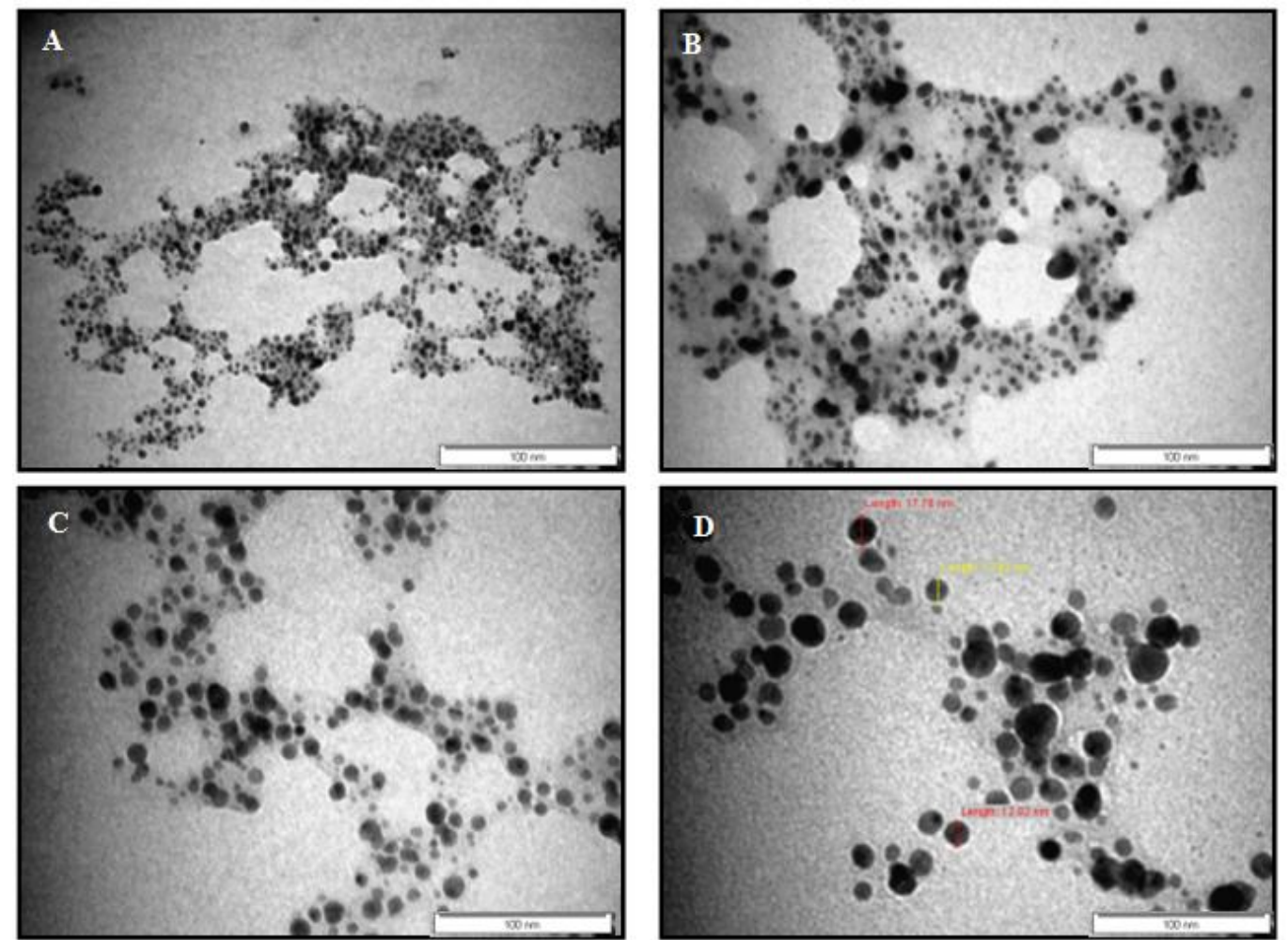

Fig. 7: The Transmission Electron Images of The Synthesised Silver Nanoparticles Using Watermelon Extract Using Different Magnifications (A) $100 \times$, (B) $150 \times$, (C) $250 \times$, and (D) $400 \times$.

The fabricated SNPs were studied using EDAX since the peaks obtained from the EDAX spectrum give the element composition of the sample. Figure 8 shows the EDAX spectrum showing strong signals for silver, which revealed that the nano-structure was formed of silver. In addition, other elements were observed on the left part of the spectrum, namely carbon (C) at $0.2 \mathrm{keV}$ and oxygen (O) at $0.5 \mathrm{keV}$. The reason for these elements appearing could be the proteins and enzymes that stabilised the nanoparticles. The same result was obtained by other groups [19].

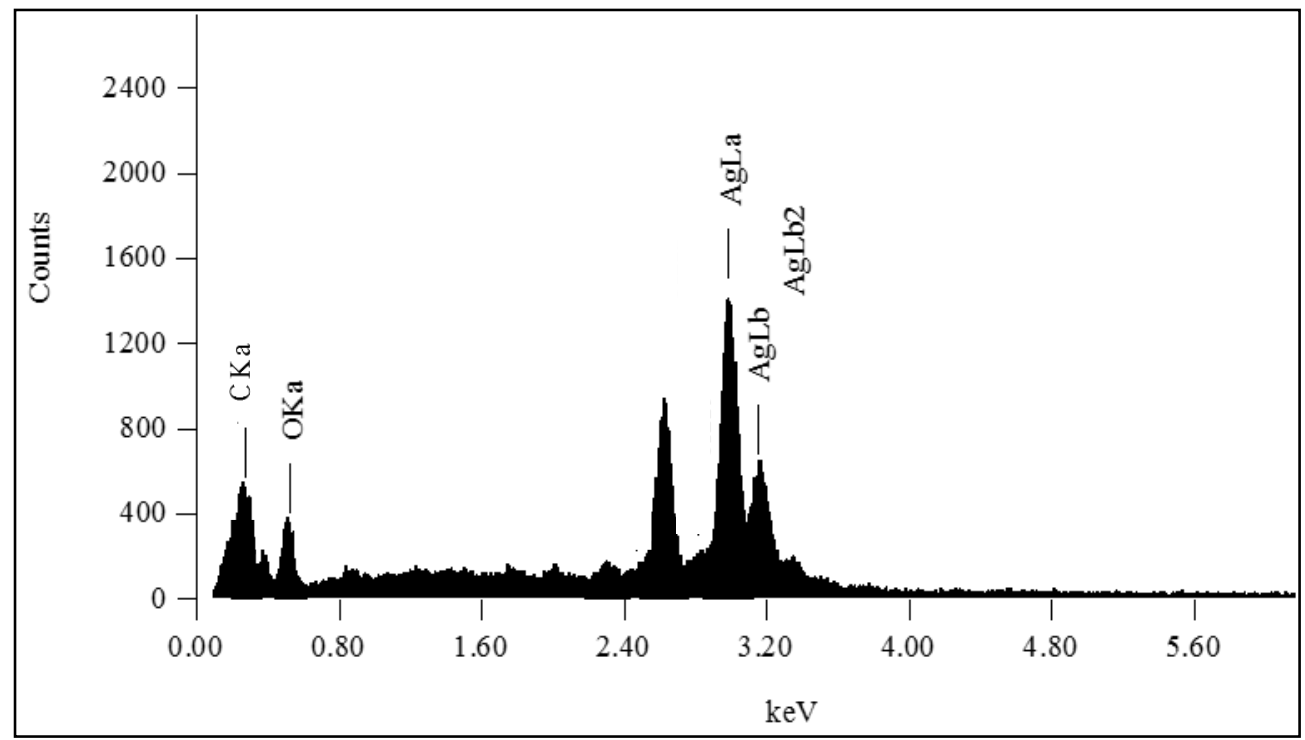

Fig. 8: EDAX Image of Snps Fabricated Using Watermelon Extract. 


\subsection{Antibacterial effect}

In this work, the antibacterial properties of the silver nanoparticles fabricated using the green route were studied using the modified disc diffusion method, which was performed in a Luria Bertani (LB) medium solid agar Petri dish. The antibacterial activity studies were performed using E. coli and S. aureus. A standard antibiotic disc (ciprofloxacin) was utilised as a reference drug while silver nitrate solution and plant extract were utilised as control. Figure 9 shows the bacterial activities of the SNPs against human pathogenic bacteria. From the figure, it can be seen that the fabricated SNPs have greatest antibacterial effect against gram-positive organisms (S. aureus) than gram-negative organisms (E. coli).
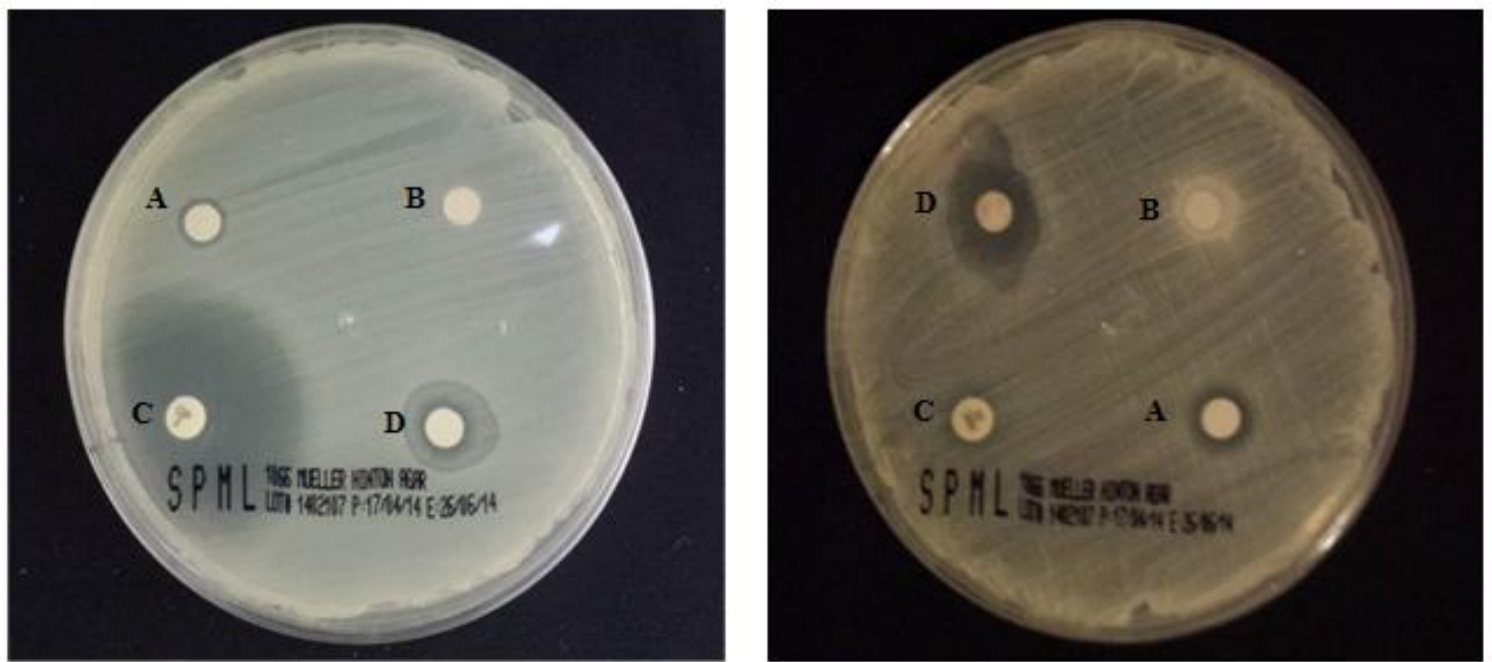

Fig. 9: Bacterial Activities of Fabricated Snps Against E. Coli (Left), and S. Aureus (Right); (A) Plant Extract, (B) Silver Nitrate Solution, (C) Antibiotic, and (D) Snps.

Table 1 presents a comparison of the activities of ciprofloxacin and silver nanoparticles on gram (-) and gram (+) bacteria. For E. coli, the zone of ciprofloxacin inhibition was $37 \mathrm{~mm}$, while the zone of inhibition of the SNPs was 16 $\mathrm{mm}$. For S. aureus, the zone of ciprofloxacin inhibition was $8 \mathrm{~mm}$, while the zone of inhibition of the SNPs was $14 \mathrm{~mm}$. These results confirm that the synthesised SNPs are effective against human pathogenic bacteria.

Table 1: Antibacterial Zone Formation by Control and Fabricated Snps against E.Coli, And S. Aureus.

\begin{tabular}{|c|c|c|}
\hline \multirow{2}{*}{ Bacteria } & \multicolumn{2}{|c|}{ Zone of inhibition (mm) } \\
\cline { 2 - 3 } & Commercial antibiotic Ciprofloxacin & Fabricated SNPs \\
\hline E. coli & $37 \pm 0.4$ & $16 \pm 0.5$ \\
\hline S. aureus & $7 \pm 0.3$ & $14 \pm 0.2$ \\
\hline
\end{tabular}

\section{Summary}

The present study demonstrated the biosynthesis of silver nanoparticles using watermelon extract. The procedure of fabrication of SNPs described in this work was simple, cost-effective, energy-saving and environment-friendly. The various parameters for optimised fabrication of silver nanoparticles were monitored using a UV-Visible spectrophotometer. The formation of the silver nanoparticles was confirmed using TEM analysis and EDAX analysis. The fabricated SNPs were found to be highly toxic against pathogenic bacteria and to exhibit antibacterial and antioxidant properties. Based on the result obtained, this effective green synthesis of silver nanoparticles could be utilised in biomedical and biotechnology applications.

\section{Acknowledgements}

This work was funded by the King Abdulaziz City for Science and Technology (KACST).

\section{References}

[1] Jain, D., et al., Synthesis of plant-mediated silver nanoparticles using papaya fruit extract and evaluation of their anti microbial activities. Digest journal of nanomaterials and biostructures, 2009. 4(3): p. 723 - 727.

[2] Murphy, C.J., Sustainability as an emerging design criterion in nanoparticle synthesis and applications. Journal of Materials Chemistry, 2008. 18(19): p. 2173-2176. http://dx.doi.org/10.1039/b717456j. 
[3] Basavaraj udapudi, B., et al., Synthesis and characterisation of silver nanoparticles. International Journal of Pharmacy and Biological Sciences, 2012. 2| (3): p. 10-14.

[4] Bar, H., et al., Green synthesis of silver nanoparticles using latex of Jatropha curcas. Colloids and Surfaces A: Physicochemical and Engineering Aspects, 2009. 339(1): p. 134-139. http://dx.doi.org/10.1016/j.colsurfa.2009.02.008.

[5] Begum, N.A., et al., Biogenic synthesis of $\mathrm{Au}$ and Ag nanoparticles using aqueous solutions of Black Tea leaf extracts. Colloids and Surfaces B: Biointerfaces, 2009. 71(1): p. 113-118. http://dx.doi.org/10.1016/j.colsurfb.2009.01.012.

[6] Shankar, S.S., et al., Rapid synthesis of Au, Ag, and bimetallic Au core-Ag shell nanoparticles using Neem (Azadirachta indica) leaf broth. Journal of Colloid and Interface Science, 2004. 275(2): p. 496-502. http://dx.doi.org/10.1016/j.jcis.2004.03.003.

[7] Prabhu, S. and E. Poulose, Silver nanoparticles: mechanism of antimicrobial action, synthesis, medical applications, and toxicity effects. International Nano Letters 2012. 2(32): p. 1-10.

[8] Geetha, N., et al., Biofabrication of Silver Nanoparticles Using Leaf Extract of Chromolaena Odorata (L.) King and Robinson. International Conference on Nuclear Energy, Environmental and Biological Sciences, 2012. 8: p. 56-59.

[9] Saxena, A., R. Tripathi, and R. Singh, biological synthesis of silver nanoparticles by using onion and their antibacterial activity. Digest Journal of Nanomaterials and Biostructures, 2010. 5(2): p. 427-432.

[10] Torresday, J.L.G., et al., Formation and growth of Au nanoparticles inside live alfa alfa plants. Nanoletters, 2004. 2(4): p. 397-401. http://dx.doi.org/10.1021/n1015673+.

[11] Bai, H.J., et al., Biosynthesis of cadmium sulfide nanoparticles by photosynthetic bacteria Rhodopseudomonas palustris. Colloids and surfaces B: Biointerfaces, 2009. 70: p. 142-146. http://dx.doi.org/10.1016/j.colsurfb.2008.12.025.

[12] Song, J.Y., H.K. Jang, and B.S. Kim, Biological synthesis of gold nanoparticles using Magnolia kobus and Diopyros kaki leaf extracts. Process Biochemistry, 2009. 44: p. 1133-1138. http://dx.doi.org/10.1016/j.procbio.2009.06.005.

[13] Elumalai, E.K., et al., Green synthesis of silver nanoparticle using Euphorbia hirta L and their antifungal activities. .Archives of Applied Science Research, 2010. 2 (6): p. 76-81.

[14] Nagajyoti, P., et al., Bio-fabrication of silver nanoparticles using leaf extract of Saururus chinenis. Digest Journal of Nanomaterials and Biostructures 2011. 6: p. 121-133.

[15] Awwad, A.M. and N.M. Salem, Green Synthesis of Silver Nanoparticles byMulberry LeavesExtract. Nanoscience and Nanotechnology 2012. 2(4): p. 125-128. http://dx.doi.org/10.5923/j.nn.20120204.06.

[16] Ponarulselvam, S., et al., Synthesis of silver nanoparticles using leaves of Catharanthus roseus Linn. G. Don and their antiplasmodial activities. Asian Pac J Trop Biomed., 2012. 2(7): p. 574-580. http://dx.doi.org/10.1016/S2221-1691(12)60100-2.

[17] kumar, D.S., Rapid and green synthesis of silver nanoparticles using leaf extracts of parthenium hysterophorus: a novel biological approach International Research Journal of Pharmacy, 2012. 3(2): p. 169-173.

[18] Prathna, T.C., et al., Biomimetic Synthesis of Nanoparticles: Science, Technology and Applicability. Biomimetics Learning from Nature. 2010, , book edited by Amitava Mukherjee, ISBN 978-953-307-025-4.

[19] Ganaie, S., et al., Biomimetic synthesis of silver nanoparticles using the amphibious weed ipomoea and their application in pollution control. Journal of King Saud University-Science, 2014. 26(3); p. 222-229. http://dx.doi.org/10.1016/j.jksus.2014.02.004. 